\title{
Proper dominating functions of graphs
}

\author{
James Hallas ${ }^{1}$, Maria Talanda-Fisher ${ }^{2}$, Ping Zhang ${ }^{2, *}$ \\ ${ }^{1}$ Department of Mathematics and Computer Science, Concord University, Athens, WV 24712, USA \\ ${ }^{2}$ Department of Mathematics, Western Michigan University, Kalamazoo, MI 49008, USA
}

(Received: 16 September 2020. Received in revised form: 30 September 2020. Accepted: 1 October 2020. Published online: 3 October 2020.)

(C) 2020 the authors. This is an open access article under the CC BY (International 4.0) license (www.creativecommons.org/licenses/by/4.0/).

\begin{abstract}
A function $f: V(G) \rightarrow\{0,1\}$ defined on the vertex set of a graph $G$ is a dominating function of $G$ if the associated function $c_{f}: V(G) \rightarrow\{0,1,2, \ldots, \Delta(G)+1\}$ defined by $c_{f}(v)=\sum_{u \in N[v]} f(u)$ has the property that $c_{f}(v) \geq 1$ for every vertex $v$ of $G$. If $c_{f}(x) \neq c_{f}(y)$ for every two adjacent vertices $x$ and $y$ of $G$, then $f$ is a proper dominating function of $G$. Sufficient conditions are obtained under which a graph has a proper dominating function and sufficient conditions are obtained under which a graph does not have a proper dominating function. For certain classes of graphs, all those members possessing a proper dominating function are determined.
\end{abstract}

Keywords: domination; dominating function; proper domination.

2020 Mathematics Subject Classification: 05C05, 05C15, 05 C69.

\section{Introduction}

A vertex $v$ in a graph $G$ is said to dominate a vertex $u$ if either $u=v$ or $u v \in E(G)$, that is, a vertex $v$ dominates the vertices in its closed neighborhood $N[v]=N(v) \cup\{v\}$. A set $S$ of vertices in $G$ is a dominating set of $G$ if every vertex of $G$ is dominated by at least one vertex in $S$. The minimum number of vertices in a dominating set of $G$ is the domination number $\gamma(G)$ of $G$. We refer to the books $[2,3,6]$ for graph theory notation and terminology not described in this paper.

There is another way that domination and the domination number of a graph $G$ have been looked at (see [2]). For a graph $G$ with maximum degree $\Delta(G)$, each function $f: V(G) \rightarrow\{0,1\}$ gives rise to another function $c_{f}: V(G) \rightarrow$ $\{0,1, \ldots, \Delta(G)+1\}$ defined by $c_{f}(v)=\sum_{u \in N[v]} f(u)$. If $c_{f}(v) \geq 1$ for every vertex $v$ of $G$, then $f$ is a dominating function of $G$. If $f$ is a dominating function of $G$, then the set $\{v \in V(G): f(v)=1\}$ is a dominating set of $G$. The domination number $\gamma(f)$ of a dominating function $f$ of a graph $G$ is $\gamma(f)=\sum_{v \in V(G)} f(v)$ and so the domination number $\gamma(G)$ of $G$ can be defined as $\gamma(G)=\min \{\gamma(f): f$ is a dominating function of $G\}$.

If $f$ is a dominating function of a graph $G$ and $c_{f}(v)$ is the same positive integer $k$ for every vertex $v$ of $G$, then $f$ is called a regular (or k-regular) dominating function of $G$. Consequently, if $G$ has a $k$-regular dominating function, then there is a dominating set $S$ of $G$ such that every vertex of $G$ is dominated by exactly $k$ vertices of $S$. Not every graph has a regular dominating function. The primary topic studied in $[4,5]$ was that of investigating graphs having a regular dominating function.

A graph $G$ has been called irregular if the vertices of $G$ have distinct degrees. It is well known that there is no nontrivial irregular graph. There is a related dominating function for graphs. A dominating function $f$ of a nontrivial graph $G$ is an irregular dominating function if $c_{f}(u) \neq c_{f}(v)$ for every two vertices $u$ and $v$ of $G$. It was observed in [4] that not only is no graph irregular, but no graph possesses an irregular dominating function.

Theorem 1.1. No nontrivial connected graph has an irregular dominating function.

Although no nontrivial graph is irregular, there are graphs with exactly two vertices having the same degree. These graphs have been referred to by many names in the literature but here we refer to them as antiregular graphs (see [1]). Formally then, a nontrivial graph $G$ is antiregular if exactly two vertices of $G$ have the same degree. For each integer $n \geq 2$, it is known that there are exactly two non-isomorphic antiregular graphs of order $n$. The two antiregular graphs of a given order $n \geq 2$ are complementary, one of which has a vertex of degree $n-1$ (and is therefore connected) and the other has an isolated vertex. A dominating function $f$ of a nontrivial graph $G$ of order $n$ is called an antiregular dominating function if there are exactly two vertices $u$ and $v$ of $G$ such that $c_{f}(u)=c_{f}(v)$. It was shown in [4] that not only does every antiregular graph have an antiregular dominating function but there are also non-antiregular graphs that have an antiregular dominating function. 
Theorem 1.2. For each integer $n \geq 4$, there are exactly $n-1$ non-isomorphic connected graphs of order $n$ having an antiregular dominating function, one graph of which is antiregular.

If one were to consider a dominating function $f$ of a graph $G$ as giving rise to a vertex coloring $c_{f}$ of $G$, then a regular dominating function results in a monochromatic vertex coloring of $G$ (all vertices of $G$ are colored the same). The most studied vertex colorings, and certainly those of greatest interest, are proper vertex colorings, however. This leads us to the following concept. If $f$ is a dominating function of a graph $G$ such that $c_{f}(u) \neq c_{f}(v)$ for each pair $u, v$ of adjacent vertices of $G$, then $f$ is a proper dominating function of $G$. For example, if $G$ is a graph in which every two adjacent vertices have different degrees, then the function that assigns 1 to every vertex of $G$ is a proper dominating function of $G$. All such graphs $G$ have the property that the set of all vertices of $G$ having a specific degree is independent. For example, if $G=K_{s, t}$ is the complete bipartite graph where $s \neq t$, then the function that assigns 1 to every vertex of $G$ is a proper dominating function of $G$. The two non-bipartite graphs in Figure 1 also have this property, where each vertex is labeled (colored) with its degree.
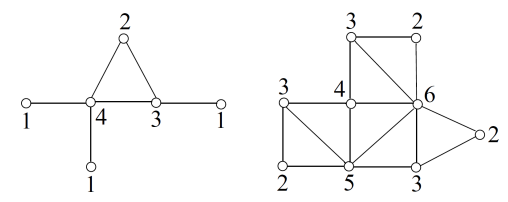

Figure 1: Two graphs in which no two adjacent vertices have the same degree.

As is the case with regular dominating functions, not every graph has a proper dominating function. There are graphs where one can see quite quickly that they do not possess a proper dominating function. Two vertices $u$ and $v$ in a connected graph $G$ are called twins if $u$ and $v$ have the same neighborhood. If $u$ and $v$ are adjacent twins in a graph $G$, then $c_{f}(u)=$ $c_{f}(v)$ for every dominating function $f$ of $G$. Thus, we have the following observation.

Observation 1.1. If a graph $G$ contains two adjacent twins, then $G$ does not have a proper dominating function.

As a consequence of Observation 1.1, it follows, for example, that no complete graph $K_{n}$ of order $n \geq 2$ has a proper dominating function. In fact, every dominating function of a complete graph is a regular dominating function. While the complete graphs $K_{n}, n \geq 2$, are regular graphs that do not have a proper dominating function, regular graphs that are not complete may or may not have a proper dominating function. For example, of the two 3-regular graphs of order 6 , the complete bipartite graph $K_{3,3}$ has a proper dominating function, while the prism $C_{3} \square K_{2}$ does not. The fact that $K_{3,3}$ has a proper dominating function is a consequence of the following result dealing with connected bipartite graphs.

Proposition 1.1. Let $G$ be a connected bipartite graph. If each vertex in one of the partite sets of $G$ has degree 2 or more, then $G$ has a proper dominating function. Consequently, if $\delta(G) \geq 2$, then $G$ has a proper dominating function.

Proof. Let $U$ and $W$ be the partite sets of $G$ such that $\operatorname{deg} u \geq 2$ for every $u \in U$. Define a function $f: V(G) \rightarrow\{0,1\}$ by $f(u)=0$ for each $u \in U$ and $f(w)=1$ for each $w \in W$. Since $c_{f}(u)=\operatorname{deg} u \geq 2$ for each $u \in U$ and $c_{f}(w)=f(w)=1$ for each $w \in W$, it follows that $f$ is a proper dominating function of $G$.

This result also shows that every grid has a proper dominating function.

Corollary 1.1. For an integer $n \geq 2$, the grid $P_{n} \square K_{2}$ has a proper dominating function.

The fact that $C_{3} \square K_{2}$ does not have a proper dominating function is a consequence of the following result. The clique number $\omega(G)$ of a graph $G$ is the maximum order of a complete subgraph of $G$.

Proposition 1.2. If $G$ is a connected graph with clique number $\omega(G) \geq k$ for some integer $k \geq 3$ and maximum degree $\Delta(G) \leq 2 k-3$, then $G$ does not have a proper dominating function.

Proof. Assume, to the contrary, that $G$ contains a complete subgraph $H$ of order $k \geq 3$ with $\Delta(G) \leq 2 k-3$ such that $G$ has a proper dominating function $f: V(G) \rightarrow\{0,1\}$. Suppose that $f$ assigns $r$ of the $k$ vertices of $H$ the value 1 . If $v \in V(H)$, then $c_{f}(v) \leq r+[\operatorname{deg} v-(k-1)] \leq r+[\Delta(G)-(k-1)] \leq r+k-2$. Consequently, $r \leq c_{f}(v) \leq r+k-2$ for every vertex $v$ of $H$. However then, two vertices of $H$ have the same $c_{f}$-value, which is impossible.

As with regular dominating functions, the primary question here is to determine which graphs (especially well-known classes of graphs) have a proper dominating function and which do not. 


\section{Which prisms have a proper dominating function?}

We saw that the prism $C_{3} \square K_{2}$ does not have a proper dominating function. We now investigate the other prisms, namely $C_{n} \square K_{2}$, where $n \geq 4$. First, we determine those cycles having a proper dominating function.

Proposition 2.1. For an integer $n \geq 3$, the cycle $C_{n}$ of order $n$ has a proper dominating function if and only if $n$ is even.

Proof. By Proposition 1.1, it remains to show that if $n$ is odd, then $C_{n}$ does not have a proper dominating function. Since $C_{3}=K_{3}$ does not have a proper dominating function, we may assume that $n \geq 5$. Let $C_{n}=\left(v_{1}, v_{2}, \ldots, v_{n}, v_{1}\right)$. Assume, to the contrary, that there is a proper dominating function $g: V\left(C_{n}\right) \rightarrow\{0,1\}$ of $C_{n}$. We claim that no two consecutive vertices of $C_{n}$ can have $g$-value 0 ; for otherwise, we may assume that $g\left(v_{1}\right)=g\left(v_{2}\right)=0$. Since $g$ is a dominating function, it follows that $g\left(v_{n}\right)=g\left(v_{3}\right)=1$. However then, $c_{g}\left(v_{1}\right)=c_{g}\left(v_{2}\right)=1$, which is a contradiction. Since $n \geq 5$ is odd and no two consecutive vertices of $C_{n}$ have $g$-value 0 , there are two consecutive vertices on $C_{n}$ having $g$-value 1 , say $g\left(v_{1}\right)=g\left(v_{2}\right)=1$. If $g\left(v_{n}\right)=g\left(v_{3}\right)$, then $c_{g}\left(v_{1}\right)=c_{g}\left(v_{2}\right)$, which is impossible. Hence, $\left\{g\left(v_{n}\right), g\left(v_{3}\right)\right\}=\{0,1\}$, say $g\left(v_{n}\right)=1$ and $g\left(v_{3}\right)=0$, which in turn forces $g\left(v_{4}\right)=1$. However then, $c_{g}\left(v_{2}\right)=c_{g}\left(v_{3}\right)=2$, which is impossible.

We now turn to prisms. We have already seen that $C_{3} \square K_{2}$ does not have a proper dominating function. This, it turns out, is the exceptional prism.

Proposition 2.2. For each integer $n \geq 4$, the prism $C_{n} \square K_{2}$ has a proper dominating function.

Proof. For each even integer $n \geq 4$, the prism $C_{n} \square K_{2}$ is a 3-regular bipartite graph and so has a proper dominating function by Proposition 1.1. Thus, it remains to show for each odd integer $n$ that $C_{n} \square K_{2}$ has a proper dominating function. Suppose that $G=C_{n} \square K_{2}$ is constructed from the two cycles $C=\left(u_{1}, u_{2}, \ldots, u_{n}, u_{1}\right)$ and $C^{\prime}=\left(v_{1}, v_{2}, \ldots, v_{n}, v_{1}\right)$ by adding the edges $u_{i} v_{i}$ for $1 \leq i \leq n$. We write $n=2 k+3$ for some positive integer $k$. Define a function $f: V(G) \rightarrow\{0,1\}$ of $G$ by $\left(f\left(u_{1}\right), f\left(u_{2}\right), \ldots, f\left(u_{n}\right)\right)=(0,1,0,1, \ldots, 0,1, \underline{1,0,1})$ and $\left(f\left(v_{1}\right), f\left(v_{2}\right), \ldots, f\left(v_{n}\right)\right)=(1,0,1,0, \ldots, 1,0, \underline{1,0,0})$, where there are $k$ pairs $(0,1)$ in the $f$-values of $C$ and $k$ pairs $(1,0)$ in the $f$-values of $C^{\prime}$. This implies that $\left(c_{f}\left(u_{1}\right), \overline{c_{f}\left(u_{2}\right)}, \ldots, c_{f}\left(u_{n}\right)\right)=$ $(3,1,3,1, \ldots, 3,1, \underline{3,2}, \underline{3,2,1})$ and $\left(c_{f}\left(v_{1}\right), c_{f}\left(v_{2}\right), \ldots, c_{f}\left(v_{n}\right)\right)=(1,3,1,3, \ldots, 1,3,1,3, \underline{2,1,2})$, where there are $k-1 \geq 0$ pairs $(3,1)$ in the $c_{f}$-values of $C$ and $k$ pairs $(1,3)$ in the $c_{f}$-values of $C^{\prime}$. Therefore, $f$ is a proper dominating function of $C_{n} \square K_{2}$ for all odd integers $n \geq 5$.

We have seen that the prism $C_{5} \square K_{2}$ has a proper dominating function. This cubic graph consists of two disjoint 5-cycles $C$ and $C^{\prime}$, where the set $\left[V(C), V\left(C^{\prime}\right)\right]$ of edges joining $C$ and $C^{\prime}$ is a perfect matching in $C_{5} \square K_{2}$. Another, even better known, cubic graph constructed in this manner is the famous Petersen graph. Before presenting a result dealing with this graph, it is useful to introduce another concept. Let $G$ be a nontrivial connected graph and let $f: V(G) \rightarrow\{0,1\}$ be a function of $G$. The complementary function $\bar{f}: V(G) \rightarrow\{0,1\}$ is defined by $\bar{f}(v)=1-f(v)$ for every vertex $v$ of $G$.

Proposition 2.3. If $f: V(G) \rightarrow\{0,1\}$ is a proper dominating function of an r-regular graph $G$ where $r \geq 2$ such that $c_{f}(v) \leq r$ for every vertex $v$ of $G$, then $\bar{f}$ is also a proper dominating function of $G$.

Proof. Observe that $c_{f}(v)+c_{\bar{f}}(v)=1+r$ for each vertex $v$ of $G$. Since $c_{f}(v) \leq r$, it follows that $c_{\bar{f}}(v) \geq 1$ and so $\bar{f}$ is a dominating function of $G$. Let $x$ and $y$ be adjacent vertices of $G$. Since $c_{f}(x) \neq c_{f}(y)$, it follows that $c_{\bar{f}}(x)=1+r-c_{f}(x) \neq$ $1+r-c_{f}(y)=c_{\bar{f}}(y)$ and so $\bar{f}$ is a proper dominating function of $G$.

Theorem 2.1. The Petersen graph does not have a proper dominating function.

Proof. Assume, to the contrary, that the Petersen graph $P$ has a proper dominating function $f: V(P) \rightarrow\{0,1\}$. Thus, $c_{f}(x) \leq 4$ for every vertex $v$ of $P$. Thus, either $c_{f}(x)=4$ for some vertex $x$ of $P$ or $1 \leq c_{f}(x) \leq 3$ for each $x \in V(P)$. We consider these two cases. We label the vertices of $P$ as shown in Figure 2.

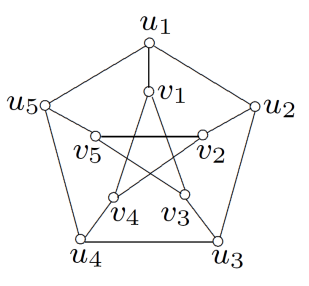

Figure 2: The Petersen graph.

Case 1. $c_{f}(x)=4$ for some vertex $x$ of $P$. Since $P$ is vertex-transitive, we may assume that $c_{f}\left(u_{1}\right)=4$. Thus, $f(x)=1$ for each $x \in N\left[u_{1}\right]$ and $2 \leq c_{f}(x) \leq 3$ for each $x \in N\left(u_{1}\right)$. 
Subcase 1.1. $c_{f}\left(u_{5}\right)=c_{f}\left(v_{1}\right)=c_{f}\left(u_{2}\right)=2$. This implies that the $f$-value of each remaining vertex of $P$ is 0 . However then, $c_{f}\left(u_{3}\right)=c_{f}\left(u_{4}\right)=1$, for example. This is a contradiction.

Subcase 1.2. Exactly two neighbors of $u_{1}$ have $c_{f}$-value 2, say $c_{f}\left(u_{5}\right)=c_{f}\left(u_{2}\right)=2$ and $c_{f}\left(v_{1}\right)=3$. Then $f(x)=0$ for each $x \in\left\{v_{2}, u_{3}, v_{5}, u_{4}\right\}$ and $\left\{f\left(v_{3}\right), f\left(v_{4}\right)\right\}=\{0,1\}$, say $f\left(v_{3}\right)=0$ and $f\left(v_{4}\right)=1$. However then, $c_{f}\left(v_{3}\right)=f\left(v_{5}\right)=1$, a contradiction.

Subcase 1.3. Exactly one neighbor of $u_{1}$ has $c_{f}$-value 2 , say $c_{f}\left(u_{5}\right)=c_{f}\left(u_{2}\right)=3$ and $c_{f}\left(v_{1}\right)=2$. Then $f\left(v_{3}\right)=f\left(v_{4}\right)=0$ and so $\left\{c_{f}\left(v_{2}\right), c_{f}\left(v_{5}\right)\right\}=\{1,2\}$, say $c_{f}\left(v_{5}\right)=1$. This implies that $f\left(v_{5}\right)=f\left(v_{2}\right)=0$ and so $c_{f}\left(v_{2}\right)=c_{f}\left(v_{5}\right)=1$, a contradiction.

Subcase 1.4. $c_{f}\left(u_{5}\right)=c_{f}\left(v_{1}\right)=c_{f}\left(u_{2}\right)=3$. Thus, $\left\{f\left(v_{3}\right), f\left(v_{4}\right)\right\}=\{0,1\}$, say $f\left(v_{3}\right)=0$ and $f\left(v_{4}\right)=1$. Thus, $c_{f}\left(u_{4}\right)=2$ or $c_{f}\left(u_{4}\right)=4$. If $c_{f}\left(u_{4}\right)=2$, then $f\left(u_{3}\right)=f\left(u_{4}\right)=0$ and so $c_{f}\left(v_{4}\right) \in\{2,3\}$, a contradiction; while if $c_{f}\left(u_{4}\right)=4$, then $f\left(u_{3}\right)=f\left(v_{4}\right)=1$ and so $c_{f}\left(u_{3}\right)=c_{f}\left(u_{2}\right)=3$, a contradiction.

Case 2. $c_{f}(x) \leq 3$ for each vertex $x$ of $P$. This implies that the complementary function $\bar{f}$ of $f$ is also a proper dominating function of $P$. Let $C=\left(u_{1}, u_{2}, u_{3}, u_{4}, u_{5}, u_{1}\right)$. We may assume that at least three vertices of $C$ have $f$-value 1 (for otherwise, we consider $\bar{f}$ ).

Subcase 2.1. Three consecutive vertices of $C$ have f-value 1 , say $f\left(u_{5}\right)=f\left(u_{1}\right)=f\left(u_{2}\right)=1$. Thus, $c_{f}\left(u_{1}\right)=3$ and so $c_{f}\left(u_{5}\right)=c_{f}\left(u_{2}\right)=2$. Thus, $f(x)=0$ for each $x \in\left\{v_{1}, v_{2}, u_{3}, u_{4}, v_{5}\right\}$. This implies that $\left\{c_{f}\left(v_{2}\right), c_{f}\left(v_{5}\right)\right\}=\{1,2\}$, a contradiction.

Subcase 2.2. No three consecutive vertices of $C$ have $f$-value 1 , say $f\left(u_{4}\right)=f\left(u_{1}\right)=f\left(u_{3}\right)=1$ and $f\left(u_{2}\right)=f\left(u_{5}\right)=0$. We may assume that $c_{f}\left(u_{4}\right)=2$ and $c_{f}\left(u_{3}\right)=3$. Thus, $f\left(v_{3}\right)=1$ and $f\left(v_{4}\right)=0$. Since $c_{f}\left(u_{5}\right)=3$, it follows that $f\left(v_{5}\right)=1$. However then, $c_{f}\left(v_{3}\right)=c_{f}\left(u_{3}\right)=3$, a contradiction.

\section{Proper dominating functions of trees}

We saw in Proposition 1.1 that if $G$ is a bipartite graph with $\delta(G) \geq 2$, then $G$ has a proper dominating function. This brings up the question of what can be said if $G$ is a bipartite graph with $\delta(G)=1$. The best known bipartite graphs with this property are trees. The following result is a consequence of Proposition 1.1.

Proposition 3.1. If $T$ is a tree such that all end-vertices of $T$ belong to the same partite set, then $T$ has a proper dominating function.

An immediate consequence of Proposition 3.1 is that every star of order 3 or more has a proper dominating function. One of the simplest classes of trees are the paths.

Proposition 3.2. For an integer $n \geq 3$, the path $P_{n}$ has a proper dominating function if and only if $n=4$ or $n$ is odd.

Proof. If $n \geq 3$ is odd, then the two end-vertices of $P_{n}$ belong to a same partite set. It then follows by Proposition 3.1 that $P_{n}$ has a proper dominating function for odd integers $n$.

For the converse, suppose that $P_{n}=\left(v_{1}, v_{2}, \ldots, v_{n}\right)$ is a path of even order $n \geq 4$. For $n=4$, the function $f$ with $f\left(v_{1}\right)=f\left(v_{2}\right)=f\left(v_{3}\right)=1$ and $f\left(v_{4}\right)=0$ is a proper dominating function. Thus, we may assume that $n \geq 6$. Assume, to the contrary, that there is a proper dominating function $g: V\left(P_{n}\right) \rightarrow\{0,1\}$ of $P_{n}$ for some even integer $n \geq 6$. First, we claim that no two consecutive vertices of $P_{n}$ can have $g$-value 0 . Since $g$ is a dominating function, it is impossible that $g\left(v_{1}\right)=g\left(v_{2}\right)=0$ or $g\left(v_{n-1}\right)=g\left(v_{n}\right)=0$. Thus, we may assume that $g\left(v_{i}\right)=g\left(v_{i+1}\right)=0$ where $2 \leq i \leq n-2$. This forces $g\left(v_{i-1}\right)=g\left(v_{i+2}\right)=1$ and so $c_{g}\left(v_{i}\right)=c_{g}\left(v_{i+1}\right)=1$, a contradiction. Necessarily, there is a vertex $v_{i}, 2 \leq i \leq n-1$, with $g\left(v_{i}\right)=0$. Thus, $g\left(v_{i-1}\right)=g\left(v_{i+1}\right)=1$, which implies that $c_{g}\left(v_{i}\right)=2$ and $c_{g}\left(v_{i-1}\right)=c_{g}\left(v_{i+1}\right)=1$. This in turns implies that $g\left(v_{i}\right)=g\left(v_{j}\right)$ if and only if $i$ and $j$ are of the same parity. We may therefore assume that $g\left(v_{i}\right)=0$ if $i$ is odd and $1 \leq i \leq n-1$ and $g\left(v_{i}\right)=1$ if $i$ is even and $2 \leq i \leq n$. However then, $c_{g}\left(v_{1}\right)=c_{g}\left(v_{2}\right)=1$, which is impossible.

A path $P_{3}=(x, y, z)$ in a connected graph $G$ of order 4 or more is called a pendant 3-path at $z$ in $G$ if $x$ is an end-vertex in $G$ and $y$ has degree 2 in $G$. In this case, $z$ is referred to as the terminal vertex of $P_{3}$. Clearly, $z$ is not an end-vertex in $G$. For example, the tree of Figure 3 has exactly three pendant 3-paths, two at the vertex $z_{1}$ and one at the vertex $z_{2}$.

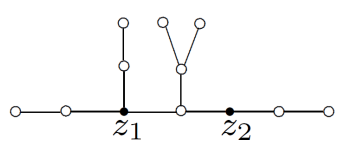

Figure 3: Illustrating pendant 3-paths and their terminal vertices.

If $P_{3}=(x, y, z)$ is a pendant 3-path at $z$ in a connected graph $G$ of at least order 4, then every proper dominating function $f$ of $G$ must assign 1 to the terminal vertex $z$; for otherwise, $c_{f}(x)=c_{f}(y)$, which is impossible. We will see that this observation is useful. We state this next. 
Observation 3.1. If $f$ is a proper dominating function of a connected graph $G$ of order 4 or more, then $f$ assigns 1 to the terminal vertex of each pendant 3-path in $G$.

We saw in Proposition 3.1 that if $T$ is a tree all of whose end-vertices of $T$ belong to the same partite set, then $T$ has a proper dominating function. The converse of Proposition 3.1 is not true, however, since the path $P_{4}$ has a proper dominating function, as stated in Proposition 3.2. In fact, there is an infinite class of trees which includes $P_{4}$ whose end-vertices belong to different partite sets and having a proper dominating function. A double star is a tree of diameter 3 . Thus, every double star $T$ has exactly two vertices that are not leaves, which are referred to as the central vertices of $T$.

\section{Proposition 3.3. Every double star has a proper dominating function.}

Proof. Let $T$ denote a double star whose central vertices are $u$ and $v$ and let $w$ be an end-vertex that is adjacent to $u$. Define a function $f: V(T) \rightarrow\{0,1\}$ by $f(u)=f(v)=f(w)=1$ and $f(x)=0$ for all remaining vertices of $T$. Since $c_{f}(u)=3$, $c_{f}(v)=c_{f}(w)=2$, and $c_{f}(x)=1$ for each $x \in V(T)-\{u, v, w\}$, it follows that $f$ is a proper dominating function.

We now consider some familiar classes of trees and determine which trees in these classes have a proper dominating function. In the first class we consider, every member possesses a proper dominating function. The non-leaf minimum degree $\delta^{*}(T)$ of a tree $T$ is the minimum degree among the non-leaves of $T$. A tree $T$ is often referred to as $r$-regular for some integer $r \geq 2$ if every non-leaf of $T$ has degree $r$. In particular, a 3-regular tree is a cubic tree. A caterpillar $T$ is a tree of order 3 or more, the removal of whose leaves produces a path called the spine of $T$. A star is therefore a caterpillar with a trivial spine and a double star is a caterpillar with spine $P_{2}$.

Proposition 3.4. Every caterpillar $T$ with $\delta^{*}(T) \geq 3$ has a proper dominating function.

Proof. Let $T$ be a caterpillar of diameter $d$. Since all stars and double stars have a proper dominating function, we may assume that $d \geq 4$. Let $\left(u_{0}, u_{1}, \ldots, u_{d-1}, u_{d}\right)$ be a path of length $d$ in $T$. We consider two cases.

Case 1 . T is a cubic caterpillar. For $1 \leq i \leq d-1$, let $v_{i}$ be the end-vertex adjacent to $u_{i}$. Define a function $f: V(T) \rightarrow$ $\{0,1\}$ of $T$ by

$$
f(x)= \begin{cases}1 & \text { if } x=u_{i} \text { for } 1 \leq i \leq d \text { or } x=v_{j} \text { for odd integer } j \text { with } 3 \leq j \leq d-1 \\ 0 & \text { otherwise. }\end{cases}
$$

It remains to show that $c_{f}$ is proper.

$\star$ If $d$ is even, say $d=2 k$ for some integer $k \geq 2$, then $\left(c_{f}\left(u_{0}\right), c_{f}\left(u_{1}\right), \ldots, c_{f}\left(u_{d}\right)\right)=(1,2,3,4,3,4, \ldots, 3,4, \underline{2})$ and $\left(c_{f}\left(v_{1}\right), c_{f}\left(v_{2}\right), \ldots, c_{f}\left(v_{d-1}\right)\right)=(\underline{1}, 1,2,1,2, \ldots, 1,2,1,2)$, where there are $k-1 \geq 0$ pairs $\overline{(3,4)}$ in the $c_{f}\left(u_{i}\right)$-values for $0 \leq i \leq d$ and $k-1$ pairs $(1,2)$ in the $c_{f}\left(v_{i}\right)$-values for $1 \leq i \leq d-1$.

$\star$ If $d$ is odd, say $d=2 k+1$ for some integer $k \geq 2$, then $\left(c_{f}\left(u_{0}\right), c_{f}\left(u_{1}\right), \ldots, c_{f}\left(u_{d}\right)\right)=(\underline{1,2}, 3,4,3,4, \ldots, 3,4, \underline{3,2})$ and $\left(c_{f}\left(v_{1}\right), c_{f}\left(v_{2}\right), \ldots, c_{f}\left(v_{d-1}\right)\right)=(\underline{1}, 1,2,1,2, \ldots, 1,2,1,2, \underline{1})$, where there are $k-1 \geq 0$ pairs $(3,4)$ in the $c_{f}\left(u_{i}\right)$-values for $0 \leq i \leq d$ and $k-1$ pairs $(1,2)$ in the $c_{f}\left(v_{i}\right)$-values for $1 \leq i \leq d-1$.

Thus, $f$ is a proper dominating function of $T$.

Case 2. $T$ is not a cubic caterpillar. Since $\delta^{*}(T) \geq 3$, it follows that $T$ contains a cubic subcaterpillar $T_{0}$ as described in Case 1 and let $f$ be the proper dominating function of $T_{0}$ defined in Case 1 . The function $f$ then can be extended to a proper dominating function $g$ of $T$ by defining $g(v)=f(v)$ if $v \in V\left(T_{0}\right)$ and $g(v)=0$ for each $v \in V(T)-V\left(T_{0}\right)$.

The condition that $\delta^{*}(T) \geq 3$ in Proposition 3.4 for a caterpillar $T$ to possess a proper dominating function is needed since $\delta^{*}(T)=2$ for the caterpillar $T$ of Figure 4 but this caterpillar has no proper dominating function. We will soon see why this tree fails to have a proper dominating function. The caterpillar $T$ of Figure 4 has the added characteristic of possessing only one vertex of degree greater than 2 . It is the class of trees containing a unique vertex of degree greater than 2 that we now consider. The simplest members of this class are the stars $K_{1, n-1}$ of order $n \geq 4$, all of which have a proper dominating function by Proposition 3.1. All other members of this class are obtained by subdividing the edges of a star of order 4 or more. As we will see, many members of this class possess a proper dominating function and many do not. We characterize all such trees possessing a proper dominating function.

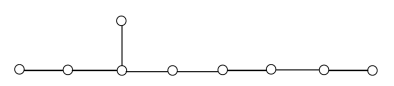

Figure 4: A caterpillar possessing no proper dominating function.

A tree $T$ is starlike if $T$ is obtained by subdividing the edges of a star of order 4 or more. Thus, the caterpillar shown in Figure 4 is a starlike tree. Equivalently, a tree $T$ is starlike if and only if $T$ has exactly one vertex whose degree is greater 
than 2. This vertex is referred to as the center of $T$. The branches of $T$ at the center are called the arms of $T$. An arm is even if its length is even; while an arm is odd if its length is odd. For example, the tree $T$ shown in Figure 5 is a starlike tree obtained by subdividing the edges of the star $K_{1,3}$. Its center is $v_{4} ; T$ has two even arms and one odd arm, one arm of each of the lengths 2,3 , and 4 .

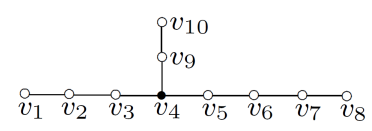

Figure 5: A starlike tree $T$.

The primary problem here is that of determining which starlike trees possess a proper dominating function. While all stars have a proper dominating function, such is not the case for starlike trees. We give one such example.

Example 3.1. The starlike tree $T$ in Figure 5 does not have a proper dominating function.

Proof. Assume, to the contrary, that $T$ has a proper dominating function $f: V(T) \rightarrow\{0,1\}$. The tree $T$ has three pendant 3-paths , namely $\left(v_{1}, v_{2}, v_{3}\right),\left(v_{10}, v_{9}, v_{4}\right)$ and $\left(v_{8}, v_{7}, v_{6}\right)$. Thus, each of the vertices $v_{3}, v_{4}, v_{6}$ is the terminal vertex of a pendant 3-path in $T$. It then follows by Observation 3.1 that $f\left(v_{3}\right)=f\left(v_{4}\right)=f\left(v_{6}\right)=1$. We consider two cases, depending on the value of $f\left(v_{5}\right)$.

Case 1. $f\left(v_{5}\right)=1$. Then $c_{f}\left(v_{5}\right)=3$, which forces that $f\left(v_{7}\right)=0$ and $f\left(v_{8}\right)=1$. However then, $c_{f}\left(v_{6}\right)=c_{f}\left(v_{7}\right)=2$, a contradiction.

Case 2. $f\left(v_{5}\right)=0$. Then $c_{f}\left(v_{5}\right)=2$. If $f\left(v_{7}\right)=1$, then $c_{f}\left(v_{5}\right)=c_{f}\left(v_{6}\right)=2$, which is impossible - necessarily then $f\left(v_{7}\right)=0$ and so $f\left(v_{8}\right)=1$. Since $c_{f}\left(v_{5}\right)=2$, it requires that $c_{f}\left(v_{4}\right)=3$ and so $c_{f}\left(v_{3}\right)=2$. Consequently, $f\left(v_{2}\right)=0$ and $f\left(v_{1}\right)=1$. However then, $c_{f}\left(v_{2}\right)=c_{f}\left(v_{3}\right)=2$, which is impossible.

The starlike tree $T$ in Example 3.1 has two even arms and one odd arm and so $T$ has arms of different parity. On the other hand, if all arms in a starlike tree have the same parity, then all end-vertices of this tree belong to the same partite set. Consequently, the following is a consequence of Proposition 3.1.

Corollary 3.1. If $T$ is a starlike tree of maximum degree 3 or more and having arms of the same parity, then $T$ has a proper dominating function.

Proposition 3.5. If $T$ is a starlike tree with diameter at most 3 , then $T$ has a proper dominating function.

Proof. Let $T$ be a starlike tree whose center is $v$ with $\operatorname{deg} v \geq 3$. Since $\operatorname{diam}(T) \leq 3$, each arm of $T$ has length at most 3. For $i=0,1,2,3$, let $V_{i}=\{w \in V(T): d(v, w)=i\}$. Thus, $V_{0}=\{v\}$. Define the function $f: V(T) \rightarrow\{0,1\}$ by $f(w)=0$ if $w \in V_{3}$ if $V_{3} \neq \emptyset$ and $f(w)=1$ if $w \notin V_{3}$. Since

$$
c_{f}(w)= \begin{cases}1 & \text { if } w \in V_{3} \\ 2 & \text { if } w \in V_{1} \text { and } w \text { is an end-vertex or } w \in V_{2} \\ 3 & \text { if } w \in V_{1} \text { and } w \text { is not an end-vertex } \\ 1+\operatorname{deg} v & \text { if } w=v .\end{cases}
$$

It follows that $f$ is a proper dominating function of $T$.

By Corollary 3.1 and Propositions 3.5, we only consider starlike trees of diameter at least 4 having arms of different parity. First, we present some useful observations. The following is a consequence of the proof of Proposition 3.2.

Observation 3.2. Let $P_{n}=\left(v_{1}, v_{2}, \ldots, v_{n}\right)$ be a path of order $n$, where $n=4$ or $n$ is odd. For a proper dominating function $f$ of $P_{n}$, let $S_{f}=\left(f\left(v_{1}\right), f\left(v_{2}\right), \ldots, f\left(v_{n}\right)\right)$.

(a) If $n=4$, then $S_{f}=(0,1,1,1)$ or $S_{f}=(1,1,1,0)$.

(b) If $n=3$, then $S_{f}=(1,1,1)$ or $S_{f}=(1,0,1)$.

(c) If $n=5$, then $S_{f}=(0,1,1,1,0)$ or $S_{f}=(1,0,1,0,1)$.

(d) If $n$ is odd and $n \neq 5$, then $S_{f}=(1,0,1,0, \ldots, 1,0,1)$.

Lemma 3.1. Let $T$ be a starlike tree whose center is $v$ with $\operatorname{deg} v \geq 3$. If $T$ has a proper dominating function $f$ such that $f(v)=0$, then each arm of $T$ has odd length or length 4 . For $u \in N(v)$, let $\ell_{u}$ be the length of the arm $A_{u}$ containing the edge vu in $T$. 
(i) If $\ell_{u}$ is odd, then $f(u)$ can be 0 or 1 when $\ell_{u}=5$ and $f(u)=1$ when $\ell_{u} \neq 5$.

(ii) If $\ell_{u}$ is even, then $\ell_{u}=4$ and $f(u)$ can be 0 or 1 .

Proof. For $u \in N(v)$, let $A_{u}=\left(v, u=v_{1}, v_{2}, \ldots, v_{\ell_{u}}\right)$ be the arm of length $\ell_{u}$ at $v$ and let $P_{\ell_{u}}=A_{u}-v=\left(v_{1}, v_{2}, \ldots, v_{\ell_{u}}\right)$. Since $f(v)=0$, the restriction of $f$ to $P_{\ell_{u}}$ is also a proper dominating function of $P_{\ell_{u}}$. It then follows by Proposition 3.2 that either $\ell_{u}$ is odd or $\ell_{u}=4$. Therefore, (i) and (ii) are consequences of Observation 3.2.

Proposition 3.6. Let $T$ be a starlike tree whose center is $v$ with $\operatorname{deg} v \geq 3$. If each arm of $T$ has odd length or length 4 , then $T$ has a proper dominating function.

Proof. For $u \in N(v)$, let $A_{u}$ be the arm of length $\ell_{u}$ at $v$ and let $P_{\ell_{u}}=A_{u}-v=\left(u=v_{1}, v_{2}, \ldots, v_{\ell_{u}}\right)$. We define a function $f: V(T) \rightarrow\{0,1\}$ as follows:

$\star$ Let $f(v)=0$.

$\star$ If $\ell_{u}=4$, let $\left(f\left(v_{1}\right), f\left(v_{2}\right), f\left(v_{3}\right), f\left(v_{4}\right)\right)=(1,1,1,0)$.

$\star$ If $\ell_{u}$ is odd, then $\left(f\left(v_{1}\right), f\left(v_{2}\right), \ldots, f\left(v_{\ell_{u}}\right)\right)=(1,0,1,0, \ldots, 1,0,1)$.

Since $f(v)=0$ and $f(u)=1$ for each $u \in N(v)$, it follows that $c_{f}(v)=\operatorname{deg} v \geq 3$ and $c_{f}(u) \in\{1,2\}$ for each $u \in N(v)$. Furthermore, the restriction of $f$ to each arm of $T$ is a proper proper dominating function of the arm. Therefore, $f$ is a proper dominating function of $T$.

Lemma 3.2. Let $T$ be a starlike tree whose center is $v$ with $\operatorname{deg} v \geq 3$. If $T$ has a proper dominating function $f$ such that $f(v)=1$, then each arm of $T$ has even length or length 1 or 3 . For $u \in N(v)$, let $\ell_{u}$ be the length of the arm $A_{u}$ containing the edge vu in $T$.

(i) If $\ell_{u}$ is even, then $f(u)$ can be 0 or 1 when $\ell_{u}=2$ and $f(u)=0$ when $\ell_{u} \neq 2$.

(ii) If $\ell_{u}=1$, then $f(u)$ can be 0 or 1 .

(iii) If $\ell_{u}=3$, then $f(u)=1$.

Proof. For $u \in N(v)$, let $A_{u}=\left(v, u=v_{1}, v_{2}, \ldots, v_{\ell_{u}}\right)$ be the arm of length $\ell_{u}$ at $v$ and let $P_{\ell_{u}}=A_{u}-v=\left(u=v_{1}, v_{2}, \ldots, v_{\ell_{u}}\right)$. Assume, to the contrary, that $\ell_{u}$ is odd and $\ell_{u} \geq 5$. Then $P_{\ell_{u}-1}=A_{u}-\{v, u\}=\left(v_{2}, v_{3}, \ldots, v_{\ell_{u}}\right)$ is a subpath of $P_{\ell_{u}}$ of even order $\ell_{u}-1 \geq 4$. We consider two cases, according to whether $f(u)=0$ or $f(u)=1$.

Case 1. $f(u)=0$. Since $f(u)=0$, the restriction of $f$ to $P_{\ell_{u}-1}$ is a proper dominating function of $P_{\ell_{u}-1}$. Since $\ell_{u}-1 \geq 4$ is even, it follows by Proposition 3.2 that $\ell_{u}-1=4$. Hence, $P_{\ell_{u}-1}=P_{4}=\left(v_{2}, v_{3}, v_{4}, v_{5}\right)$. By Observation 3.2, either $\left(f\left(v_{2}\right), f\left(v_{3}\right), f\left(v_{4}\right), f\left(v_{5}\right)\right)=(0,1,1,1)$ or $\left(\left(f\left(v_{2}\right), f\left(v_{3}\right), f\left(v_{4}\right), f\left(v_{5}\right)\right)=(1,1,1,0)\right.$, as indicated below. In either case, $c_{f}\left(v_{1}\right)=$ $c_{f}\left(v_{2}\right)$, which is impossible.

Case 2. $f(u)=1$. First, suppose that $f\left(v_{2}\right)=0$ and so $c_{f}(u)=c_{f}\left(v_{1}\right)=2$. This implies that $f\left(v_{3}\right)=0$ and so $A_{u}$ has two adjacent vertices whose $f$-value is 0 , which is impossible. Next, suppose that $f\left(v_{2}\right)=1$ and so $c_{f}(u)=c_{f}\left(v_{1}\right)=3$. This forces $f\left(v_{3}\right)=0$ and results in $c_{f}\left(v_{2}\right)=2$. Since $c_{f}\left(v_{3}\right) \neq 2$, this forces $f\left(v_{4}\right)=0$, once again, resulting in two adjacent vertices whose $f$-value is 0 , an impossibility.

The statements (i), (ii), and (iii) are then consequences of Observation 3.2.

The starlike tree $T$ in Figure 5 has two arms of length at most 3. As we saw, $T$ has no proper dominating function.

Proposition 3.7. Let $T$ be a starlike tree whose center is $v$ with $\operatorname{deg} v \geq 3$. If each arm of $T$ has even length or length 1 or 3 and at least three arms of length at most 3 , then $T$ has a proper dominating function.

Proof. For $u \in N(v)$, let $A_{u}$ be the arm of length $\ell_{u}$ at $v$ and let $P_{\ell_{u}}=A_{u}-v=\left(u=v_{1}, v_{2}, \ldots, v_{\ell_{u}}\right)$. We define a function $f: V(T) \rightarrow\{0,1\}$ as follows:

$\star$ Let $f(v)=1$.

$\star$ If $\ell_{u}=1$, let $f(u)=f\left(v_{1}\right)=1$.

$\star$ If $\ell_{u}=2$, let $\left(f\left(v_{1}\right), f\left(v_{2}\right)\right)=(1,1)$.

$\star$ If $\ell_{u}=3$, let $\left(f(u)=f\left(v_{1}\right), f\left(v_{2}\right), f\left(v_{3}\right)\right)=(1,1,0)$. 
$\star$ If $\ell_{u}$ is even, then $\left(f\left(v_{1}\right), f\left(v_{2}\right), \ldots, f\left(v_{\ell_{u}}\right)\right)=(0,1,0,1, \ldots, 0,1)$.

Since $f(v)=f(u)=1$ for each $u \in N(v)$ such that $1 \leq \ell_{u} \leq 3$ and $T$ has at least three arms of length at most 3 , it follows that $c_{f}\left(v_{i}\right) \in\{1,2,3\}$ for $1 \leq i \leq \ell_{u}$.

$$
\begin{aligned}
& \text { If } \ell_{u}=1 \text {, then } c_{f}(u)=c_{f}\left(v_{1}\right)=2 . \\
& \text { If } \ell_{u}=2 \text {, then }\left(c_{f}(u)=c_{f}\left(v_{1}\right), c_{f}\left(v_{2}\right)\right)=(3,2) . \\
& \text { If } \ell_{u}=3 \text {, then }\left(c_{f}(u)=c_{f}\left(v_{1}\right), c_{f}\left(v_{2}\right), c_{f}\left(v_{3}\right)\right)=(3,2,1), \\
& \text { If } \ell_{u} \geq 4 \text { is even, then }\left(c_{f}(u)=c_{f}\left(v_{1}\right), c_{f}\left(v_{2}\right), \ldots, c_{f}\left(v_{\ell_{u}}\right)=(2,1,2,1, \ldots, 2,1) .\right.
\end{aligned}
$$

Therefore, $f$ is a proper dominating function of $T$.

Proposition 3.8. Let $T$ be a starlike tree whose center is $v$ with $\operatorname{deg} v \geq 3$. If each arm of $T$ has even length or length 1 , at least two of which have length 1 or 2, then $T$ has a proper dominating function.

Proof. For $u \in N(v)$, let $A_{u}$ be the arm of length $\ell_{u}$ at $v$ and let $P_{\ell_{u}}=A_{u}-v=\left(u=v_{1}, v_{2}, \ldots, v_{\ell_{u}}\right)$. We define a function $f: V(T) \rightarrow\{0,1\}$ as follows:

$\star$ Let $f(v)=1$.

$\star$ If $\ell_{u}=1$, let $f(u)=f\left(v_{1}\right)=1$.

$\star$ If $\ell_{u}=2$, let $\left(f\left(v_{1}\right), f\left(v_{2}\right)\right)=(1,0)$.

$\star$ If $\ell_{u} \geq 4$, then $\left(f\left(v_{1}\right), f\left(v_{2}\right), \ldots, f\left(v_{\ell_{u}}\right)\right)=(0,1,0,1, \ldots, 0,1)$.

Since $f(v)=f(u)=1$ for each $u \in N(v)$ such that $1 \leq \ell_{u} \leq 2$ and $T$ has at least two arms of length 1 or 2 , it follows that $c_{f}\left(v_{i}\right) \in\{1,2\}$ for $1 \leq i \leq \ell_{u}$. If $\ell_{u}=1$, then $c_{f}(u)=c_{f}\left(v_{1}\right)=2$. If $\ell_{u}=2$, then $\left(c_{f}(u)=c_{f}\left(v_{1}\right), c_{f}\left(v_{2}\right)\right)=(2,1)$. If $\ell_{u} \geq 4$, then $\left(c_{f}(u)=c_{f}\left(v_{1}\right), c_{f}\left(v_{2}\right), \ldots, c_{f}\left(v_{\ell_{u}}\right)=(2,1,2,1, \ldots, 2,1)\right.$. Therefore, $f$ is a proper dominating function of $T$.

We begin with those starlike trees having at least three arms of length at most 3.

Theorem 3.1. Let $T$ be a starlike tree whose center is $v$ with $\operatorname{deg} v \geq 3$ and diameter at least 4 such that $T$ has arms of different parity and at least three arms of length 1, 2 or 3 . Then $T$ has a proper dominating function if and only if

(i) each arm of $T$ has odd length or length 4 or

(ii) each arm of $T$ has even length or length 1 or 3.

Proof. By Propositions 3.6 and 3.7, it remains for such starlike trees only to verify the necessity of this statement. Suppose that $T$ satisfies neither of (i) nor (ii). This implies that $T$ has an even arm whose length is not 4 and an arm of odd length 5 or more. We show that $T$ does not have a proper dominating function. Assume, to the contrary, that $T$ has a proper dominating function $f: V(T) \rightarrow\{0,1\}$. Since $T$ has an even arm whose length is not 4, it follows by Lemma 3.1 that $f(v)=1$. However then, all odd arms of $T$ have length 1 or 3 by Lemma 3.2, which is a contradiction.

We now consider those starlike trees without any arm of length at most 3.

Theorem 3.2. Let $T$ be a starlike tree whose center is $v$ with $\operatorname{deg} v \geq 3$ and diameter at least 4 such that $T$ has arms of different parity and no arm has length 1, 2, or 3. Then T has a proper dominating function if and only if each arm of $T$ has odd length or length 4.

Proof. By Proposition 3.6, if each arm of $T$ has odd length or length 4, then $T$ has a proper dominating function. For the converse, suppose that $T$ has an even arm whose length is not 4 and assume, to the contrary, that $T$ has a proper dominating function $f: V(T) \rightarrow\{0,1\}$. By Lemma 3.1, $f(v)=1$. However then, all odd arms of $T$ have length 1 or 3 by Lemma 3.2 , which is a contradiction.

Next, we consider those starlike trees having exactly one arm of length at most 3 . We begin with starlike trees having exactly one arm of length 1 and all other arms have length 4 or more.

Proposition 3.9. Let $T$ be a starlike tree whose center is $v$ with $\operatorname{deg} v \geq 3$ and having arms of different parity. Suppose that $T$ has exactly one arm of length 1 with all other arms of (even or odd) length 4 or more. Then $T$ has a proper dominating function if and only if all even arms of $T$ have length 4. 
Proof. Let $x \in N(v)$ such that $A_{x}=(v, x)$ has length 1. Thus, for each $u \in N(v)$, the arm $A_{u}$ at $v$ has length $\ell_{u} \geq 4$. By Proposition 3.6, if all even arms of $T$ have length 4, then $T$ has a proper dominating function. For the converse, suppose that $T$ has an even arm of length 6 or more and a proper dominating function $f: V(T) \rightarrow\{0,1\}$. By Lemma 3.1, $f(v)=1$. It then follows by Lemma 3.2 that $A_{x}$ is the only odd arm in $T$ and $f(u)=0$ for $u \in N(v)-\{x\}$. However then, $c_{f}(v)=c_{f}(x)$, which is impossible.

The situation is the same if a starlike tree has exactly one arm of length 3 and all other arms have length at least 4 .

Proposition 3.10. Let $T$ be a starlike tree whose center is $v$ with $\operatorname{deg} v \geq 3$ and having arms of different parity. Suppose that $T$ has exactly one arm of length 3 with all other arms of (even or odd) length 4 or more. Then $T$ has a proper dominating function if and only if all even arms of $T$ have length 4.

Proof. Let $x \in N(v)$ such that $A_{x}=\left(v, x=x_{1}, x_{2}, x_{3}\right)$ has length 3. Thus, for each $u \in N(v)$, the arm $A_{u}$ at $v$ has length $\ell_{u} \geq 4$. By Proposition 3.6, if all even arms of $T$ have length 4, then $T$ has a proper dominating function. For the converse, suppose that $T$ has an even arm of length 6 or more and a proper dominating function $f: V(T) \rightarrow\{0,1\}$. By Lemma 3.1, $f(v)=1$. It then follows by Lemma 3.2 that $A_{x}$ is the only odd arm in $T$ and $f(x)=1$ and $f(u)=0$ for each $u \in N(v)-\{x\}$. Let $u \in N(v)-\{x\}$. Since no two adjacent vertices on an arm (different from $v$ ) can both have $f$-value 0 , there exists a neighbor $v^{\prime}$ of $u$ distinct from $v$ such that $f\left(v^{\prime}\right)=1$. However then, $c_{f}(v)=c_{f}(u)=2$, which is impossible.

The situation is completely different for a starlike tree having arms of different parity, some of which have length 2 and others have length 4 or more.

Proposition 3.11. Let $T$ be a starlike tree whose center is $v$ with $\operatorname{deg} v \geq 3$ and having arms of different parity. If $T$ has an arm of length 2 with all other arms of (even or odd) length 4 or more, then Thas no proper dominating function.

Proof. Let $x \in N(v)$ such that the arm $A_{x}$ has length 2. Thus $A_{x}$ is a pendant $P_{3}$ at $v$. Assume, to the contrary, that $T$ has a proper dominating function $f: V(T) \rightarrow\{0,1\}$. Then $f(v)=1$. It follows by Lemma 3.2 that each odd arm of $T$ must have length 1 or 3 , which is impossible.

The following is a consequence of the proof of Proposition 3.11 (or Lemma 3.2).

Corollary 3.2. Let $T$ be a starlike tree whose center is $v$ with $\operatorname{deg} v \geq 3$ and having arms of different parity. If $T$ has an arm of length 2 and $T$ has a proper dominating function, then every odd arm of T has length 1 or 3.

First, we determine which starlike trees having arms of different parity and an arm of length 2 possess a proper dominating function.

Theorem 3.3. Let $T$ be a starlike tree whose center is $v$ with $\operatorname{deg} v \geq 3$ and having arms of different parity and an arm of length 2. Then $T$ has a proper dominating function if and only if $T$ satisfies the following conditions $(a)$ and $(b)$ :

(a) each odd arm of $T$ has length 1 or 3 and

(b) if $T$ has an arm of length 3 , then $T$ has at least three arms of length 1,2 , or 3.

Proof. First, suppose that $T$ satisfies (a) and (b). If each odd arm of $T$ has length 1, then $T$ has at least two arms of length at most 2 and so $T$ has a proper dominating function by Proposition 3.8. If $T$ has an arm of length 3 , then $T$ has at least three arms of length 1, 2, or 3 and so $T$ has a proper dominating function by Proposition 3.7.

For the converse, suppose that $T$ does not satisfy (a) or does not satisfy (b) and has a proper dominating function $f: V(T) \rightarrow\{0,1\}$. By Corollary 3.2, each arm of $T$ has even length or length 1 or length 3 . So, (a) is satisfied and (b) is not satisfied. If $T$ does not have an arm of length 3 , then (b) is true vacuously. Hence, $T$ has an arm of length 3 but $T$ has at most two arms of length 1, 2 or 3 . Since $T$ has an arm of length 2 , it follows that $T$ has exactly one odd arm and this odd arm has length 3. Therefore, $T$ has exactly one arm of length 2, exactly one arm of length 3 , and all other arms have even length 4 or more. Let $x, y \in N(v)$ such that the length of $A_{x}$ is 2 and the length of $A_{y}$ is 3 , say $A_{x}=\left(v, x, x^{\prime}\right)$ and $A_{y}=\left(v, y, y^{\prime}, y^{\prime \prime}\right)$. For each $u \in N(v)-\{x, y\} \neq \emptyset$, the arm $A_{u}$ has even length 4 or more, say $A_{u}=\left(v, u=v_{1}, v_{2}, \ldots, v_{\ell_{u}}\right)$ where $\ell_{u}$ is even and $\ell_{u} \geq 4$. By Lemma 3.2, it follows that $\left(f(v), f(x), f\left(x^{\prime}\right)\right) \in\{(1,0,1),(1,1,0),(1,1,1)\},\left(f(v), f(y), f\left(y^{\prime}\right), f\left(y^{\prime \prime}\right)\right)=(1,1,1,0)$ and $\left(f(v), f\left(v_{1}\right), f\left(v_{2}\right), \ldots, f\left(v_{\ell_{u}}\right)\right)=(1,0,1,0,1, \ldots, 0,1)$. Thus, $c_{f}(x) \in\{2,3\}, c_{f}(y)=3$ and $c_{f}(u)=2$. If $f(x)=0$, then $c_{f}(v)=c_{f}(u)=2$; while if $f(x)=1$, then $c_{f}(v)=c_{f}(y)=3$. In either case, a contradiction is produced.

We now consider starlike trees having arms of different parity but no arm of length 2 . In order to do this, we first present a result that is similar to Propositions 3.9 and 3.10 . 
Proposition 3.12. Let $T$ be a starlike tree whose center is $v$ with $\operatorname{deg} v \geq 3$ and having arms of different parity. Suppose that $T$ has two arms of length 1 or 3 , at least one of which has length 3, with all other arms of (even or odd) length 4 or more. Then $T$ has a proper dominating function if and only if all even arms of $T$ have length 4.

Proof. Let $x, y \in N(v)$ such that $A_{x}$ has length 1 or 3 and $A_{y}=\left(v, y=y_{1}, y_{2}, y_{3}\right)$ has length 3 . If all even arms of $T$ have length 4, then $T$ has a proper dominating function by Proposition 3.6. For the converse, suppose that $T$ has an even arm of length 6 or more and a proper dominating function $f: V(T) \rightarrow\{0,1\}$. By Lemma 3.1, $f(v)=1$. It then follows by Lemma 3.2 that (i) $A_{x}$ and $A_{y}$ are the only odd arms in $T$ and (ii) $f(y)=1$ and $f(u)=0$ for each $u \in N(v)-\{x, y\} \neq \emptyset$. For $u \in N(v)-\{x, y\}$, let $A_{u}=\left(v, u=v_{1}, v_{2}, \ldots, v_{\ell_{u}}\right)$ where then $\ell_{u}$ is even and $\ell_{u} \geq 4$. Since $f(u)=f\left(v_{1}\right)=0$ and no two adjacent vertices on an arm (different from $v$ ) can both have $f$-value 0 , it follows that $f\left(v_{2}\right)=1$ and so $c_{f}(u)=2$. This forces $f(x)=1$ and so $c_{f}(v)=3$. If $f\left(y_{2}\right)=1$, then $c_{f}(v)=c_{f}(y)=3$, while if $f\left(y_{2}\right)=0$, then $f\left(y_{3}\right)=1$ and so $c_{f}(y)=c_{f}\left(y_{2}\right)=2$, which is impossible in either case.

Theorem 3.4. Let $T$ be a starlike tree whose center is $v$ with $\operatorname{deg} v \geq 3$ and having arms of different parity but no arms of length 2. Then $T$ has a proper dominating function if and only if $T$ satisfies one of the following conditions:

(i) Each even arm of $T$ has length 4.

(ii) Some even arm of $T$ has length 6 or more and each odd arm has length 1 or 3 such that either $T$ has at least two arms of length 1 or $T$ has at least three odd arms, each of which has length 1 or 3.

Proof. If $T$ satisfies (i), then $T$ has a proper dominating function by Proposition 3.6. If $T$ satisfies (ii), then $T$ has a proper dominating function by Proposition 3.8 and Theorem 3.1.

For the converse, suppose that $T$ satisfies neither (i) nor (ii), but $T$ has a proper dominating function $f: V(T) \rightarrow\{0,1\}$. Since $T$ does not satisfy (i), it follows that $T$ has an even arm of length 6 or more. Let $x \in N(v)$ such that $A_{x}$ is an even arm of length $\ell_{x} \geq 6$. It then follows by Lemma 3.1 that $f(v)=1$ and so $f(x)=0$ by Lemma 3.2(i). Since $f(v)=1$, it follows by Lemma 3.2 that each odd arm of $T$ has length 1 or 3 . Since $T$ does not satisfy (ii), it follows that $T$ has one or two odd arms, each of which has length 1 or 3 and at least one of which has length 3 . However then, all even arms must have length 4 by Propositions 3.9, 3.10, and 3.12. This contradicts the assumption that $T$ has an even arm of length 6 or more.

By Corollary 3.1, Propositions 3.5 and 3.8, and Theorems 3.3 and 3.4, we are now prepared to present a characterization of all starlike trees $T$ with maximum degree 3 or more such that $T$ has a proper dominating function.

Theorem 3.5. A starlike tree $T$ with maximum degree 3 or more has a proper dominating function if and only if $T$ satisfies any of the following conditions:

(a) The diameter of $T$ is at most 3.

(b) All arms of $T$ have the same parity.

(c) Each arm of $T$ has odd length or length 4.

(d) Some even arm of $T$ has length 2 or at least length 6 and each odd arm has length 1 or 3 such that either $T$ has at least two arms of length at most 2 or $T$ has at least three arms of length at most 3 ,

\section{Acknowledgments}

We are grateful to Professor Gary Chartrand for suggesting the concept of proper dominating functions to us and kindly providing useful information on this topic. We also thank the anonymous referees whose valuable suggestions resulted in an improved paper.

\section{References}

[1] A. Ali, G. Chartrand, P. Zhang, Irregularity in Graphs, Springer, New York, In press.

[2] G. Chartrand, T. W. Haynes, M. A. Henning, P. Zhang, From Domination to Coloring: Stephen Hedetniemi's Graph Theory and Beyond, Springer, New York, 2019.

[3] G. Chartrand, P. Zhang, Chromatic Graph Theory, Second Edition, Chapman \& Hall/CRC Press, Boca Raton, 2020.

[4] J. Hallas, M. Talanda-Fisher, P. Zhang, Dominating functions in graphs-regularity versus irregularity. Int. J. Comput. Math. Comput. Systems Theory 5 (2020) 98-111.

[5] J. Hallas, M. Talanda-Fisher, P. Zhang, Regular dominating functions in regular graphs, Bull. Inst. Combin. Appl. 90 (2020) $139-157$.

[6] T. W. Haynes, S. T. Hedetniemi, P. J. Slater, Fundamentals of Domination in Graphs, Marcel Dekker, New York, 1998.

[7] T. W. Haynes, S. T. Hedetniemi, P. J. Slater (Eds), Domination in Graphs: Advanced Topics, Marcel Dekker, New York, 1998.

[8] O. Ore, Theory of Graphs, American Mathematical Society Colloquium Publications, Providence, 1962. 